\title{
Suicide ideation, plans, and attempts among general practice patients with chronic health conditions in Puerto Rico
}

This article was published in the following Dove Press journal:

International Journal of General Medicine

10 March 2011

Number of times this article has been viewed

\author{
Mildred Vera ${ }^{2,4}$ \\ María L Reyes-Rabanillo' \\ Sarah Huertas ${ }^{3}$ \\ Deborah Juarbe ${ }^{4}$ \\ Coralee Pérez-Pedrogo ${ }^{4}$ \\ Aracelis Huertas ${ }^{5}$ \\ Marisol Peña ${ }^{6}$ \\ 'Veterans Affairs Caribbean \\ Healthcare System, San Juan, Puerto \\ Rico; ${ }^{2}$ Department of Health Services \\ Administration, School of Public \\ Health; ${ }^{3}$ Department of Psychiatry, \\ School of Medicine; ${ }^{4}$ Center for \\ Evaluation and Sociomedical Research, \\ School of Public Health; ${ }^{5} \mathrm{School}$ \\ of Health Professions; ${ }^{6}$ Center for \\ Preparedness in Public Health, School \\ of Public Health, Medical Sciences \\ Campus, University of Puerto Rico \\ San Juan, Puerto Rico.
}

Correspondence: Mildred Vera University of Puerto Rico, Medical Sciences Campus, School of Public Health, PO Box 365067, San Juan, Puerto Rico 00936-5067

Tel +17877582525 ext 1422

Fax +787 7674146

Email mildred.vera@upr.edu
Background: Little is known about suicidal ideation among general practice patients in Puerto Rico. In this study we examined the rates, severity, and correlates of suicidal ideation, plans, and attempts among general practice patients with chronic illnesses. This is important in targeting appropriate interventions and management approaches to minimize and prevent suicide.

Methods: We screened patients with chronic physical conditions at general practices. Suicidal ideation was assessed with the suicidality module of the Mini International Neuropsychiatric Interview. Major depression was assessed with the Patient Health Questionnaire depression module. The relationship between sociodemographic factors, depression and suicidal ideation was examined with multiple logistic regression analysis. Among the subgroup that acknowledged suicidal ideation, we used multinomial logistic regression analysis to estimate simultaneously the multivariate associations of depression and sociodemographic factors with suicidality risk levels.

Results: Of the 2068 patients screened, 15.4\% acknowledged recent suicidal ideation. Among this group, $8.6 \%$ reported passive ideation, $3.7 \%$ active ideation without a plan, and $3.1 \%$ active ideation with a plan or attempt. According to multivariate logistic regression, suicidal ideation was higher among patients with moderately severe depression and severe depression than for those with milder symptoms. Patients aged 64 years or younger were over one and a half times more likely to acknowledge suicidal ideation than those aged 65 years and older. Compared with patients having a college degree, those with lower education had a twofold higher risk of suicidal ideation. Multinomial logistic regression analysis indicated that severe depression was associated with a higher likelihood of having a suicide plan or attempt.

Conclusion: The findings of this study suggest that public health strategies focusing on the systematic identification of patients with increased depression severity and the implementation of evidence-based depression treatments are relevant for minimizing and preventing suicidal behavior among general practice patients with chronic health conditions.

Keywords: suicidal ideation, chronic illnesses, depression, Puerto Rican

\section{Introduction}

Worldwide, approximately one million people die from suicide each year. ${ }^{1}$ In Puerto Rico, a Caribbean island of almost four million inhabitants, suicide is among the top 15 causes of death, claiming approximately 3000 lives over the past decade. Suicide attempts are even more common, and for every completed suicide, there are 8-25 times as many suicide attempts. ${ }^{2}$ The World Health Organization has acknowledged that suicidal behavior is a major public health problem in every country, highlighting 
the relevance of effective preventive strategies that target the reduction of deaths by suicide. ${ }^{3,4}$

Depression, one of the most common mental disorders seen in general medical care, is a significant risk factor for suicidal behaviors. ${ }^{5,6}$ While major depressive disorder accounts for about $20 \%-35 \%$ of all suicide deaths, a substantial number of people who commit suicide fail to receive appropriate treatment. ${ }^{7}$ Data from psychological autopsy studies show that only $6 \%-14 \%$ of depressed suicide victims received adequate treatment, although the possibility of delivering care had existed, because $50 \%-70 \%$ of those who completed suicide had visited a nonmental health clinician in the month prior to their death. ${ }^{8}$ Given that at the present time it is not possible to predict accurately who will commit suicide, it has been stated that the recognition, and consequently the treatment of depression in general medical settings deserves more attention. ${ }^{4,8}$

Findings from general practice patient samples suggest that chronic illness is another significant risk factor for both suicidal ideation and suicide attempts. ${ }^{9-12}$ Chronic illness is estimated to be involved in up to $25 \%$ of all suicides. ${ }^{2}$ Investigators have found high rates of depression in patients with chronic illnesses, suggesting that depression may have a mediating role in the relationship between chronic illness and suicidal behavior. ${ }^{13,14}$ Patients with depression are at high risk for suicidal ideation, and patients with depression and comorbid chronic medical conditions, are at especially high risk.

Suicidal risk level is associated with a greater likelihood of suicide completion. ${ }^{15}$ Suicidal behavior is often seen as a continuum extending from wishing to be dead or passive ideation, thoughts and plans about harming oneself, suicide attempts and completed suicide. ${ }^{16}$ The significance of suicidal risk varies from less severe (thoughts of death) to highest severity (suicidal ideation with plan or recent suicidal attempt). ${ }^{17}$ It has been suggested that to identify patients at increased risk for suicide it is necessary to obtain more precise knowledge about suicidality risk levels and related predictors. ${ }^{18}$

Most studies that have examined suicidal ideation and suicidality risk levels in general practice patients have included few or no Puerto Ricans at all. Consequently, little is known about suicidality among Puerto Rican general medical patients. Toward this end, in this study we examined the rate and correlates of suicidal ideation and suicidality risk levels among general practice patients with chronic illnesses in Puerto Rico. Our overall goal was to gain a better understanding of the risk or protective factors separating passive suicidal ideation from more serious ideation. This is important in targeting appropriate interventions and management approaches to minimize and prevent suicide.

\section{Methods}

The present study reports data from the Collaborative Care in General Medical Settings study. ${ }^{19}$ The main aim of this study was to examine the impact of a collaborative care intervention for the management of depression, among general practice patients in Puerto Rico. Practices were located in the greater metropolitan area of San Juan, Puerto Rico, and served primarily low income patients covered by government health insurance. Health services delivery across sites had a high uniformity because government health insurance followed a model of managed care, in which the general medical sector was responsible for providing physical health care, while the provision of all mental health care was carved out to managed behavioral health organizations. A convenience sample of 2068 patients presenting for visits at 14 general practices were screened. Trained research assistants approached patients who were in clinic waiting rooms. The large number of patients in most clinics made it logistically impossible to reach patients in a systematic and timely manner. Patients were selected on the basis of availability at the moment. Over $85 \%$ of the patients approached agreed to participate. Arrangements were made with nursing personnel to guarantee that participants did not miss their clinic appointment as a result of participating in the screening. Although patients were provided this information, concerns about missing their appointment was the main reason for refusal. Interviews were conducted in private offices to ensure confidentiality.

Patients were eligible if they were 18 years or older and reported having any of the following health conditions: diabetes, hypothyroidism, hypertension, asthma, chronic bronchitis, arthritis, heart disease, high cholesterol, or stroke. They were excluded if they had a history of psychotic or bipolar disorder, were currently abusing drugs or alcohol, were cognitively impaired, did not speak Spanish, or reported that they did not intend to receive care in the clinic within the following six-month period. Those currently receiving mental health treatment or applying for disability benefits were also excluded. Screening personnel were provided special guidelines to inform the physician when patients reported life-ending plans. These patients were determined to be at high risk for suicide and were thus ineligible. All participants gave written informed consent. The study protocol was approved by the Institutional Review Board of the Medical Sciences Campus, University of Puerto Rico. 
We determined suicidal ideation with a two-tier process. The first tier screener consisted of the suicide item of the Patient Health Questionnaire depression module (PHQ-9) inquiring about thoughts of being better off dead or hurting themselves in some way during the past two weeks. ${ }^{20}$ Patients who reported death or suicidal thoughts several days or more were screened into the suicidality module of the Mini International Neuropsychiatric Interview. ${ }^{21}$ The questions were: "In the past month did you: 1) think that you would be better off dead or wish you were dead? 2) want to harm yourself? 3) think about suicide? 4) have a suicide plan? and 5) attempt suicide?". Patients endorsing any question in this section were classified as suicide ideators. Among this group, we categorized patients into four mutually exclusive subgroups: passive ideation (yes to question 1), active ideation without a plan (yes to question 2 or 3), active ideation with a plan (yes to question 4), and suicide attempt (yes to question 5).

The presence and severity of major depression was assessed with the PHQ-9. This module is a brief diagnostic instrument structured to assess Diagnostic and Statistical Manual of Mental Disorders, 4th Edition criteria for major depressive disorder. Each of the items asks the patients to indicate the frequency with which they experienced the depressive symptom during the two weeks before the interview. Each item is scored on a scale from 0 , not at all, to 3 , nearly every day. The algorithm and severity scale developed and validated by Spitzer et al was used. ${ }^{20}$ Scores from 5 to 14 indicate mild-moderate depression, scores from 15 to 19 indicate moderately severe depression, and scores of 20 and above indicate severe depression. ${ }^{22}$ The Spanish-translated PHQ-9 has good internal consistency (Cronbach's $\alpha .80$ ) and measures a concept of depression that is statistically similar to that in the English language PHQ-9. ${ }^{23}$ Sociodemographic information including age, gender, marital and employment status, and education were also obtained.

Initially, we examined bivariate relationships among study variables with Chi-square analysis for categorical data and $t$-tests for continuous indices. Next, we conducted two broad analyses. The first compared the nonideators with the ideators. The second was a subgroup analysis of patients with any suicidal ideation, comparing passive ideators without a plan, active ideators without a plan, and those with a suicidal plan or attempt in the past month. Multiple logistic regression analysis was conducted to examine the relationship between the explanatory factors and any suicidal ideation. Among the subgroup of suicide ideators, we used multinomial logistic regression to estimate simultaneously the multivariate associations of the three levels of suicidal ideation (passive ideation, active ideation, and ideation with plan or attempt) with the explanatory factors. In this analysis, passive ideation was used as the reference category and the others were compared with it. Explanatory factors that were associated with suicidal ideation included gender, age, education, marital status, employment, and depression severity. Similar to other studies that have looked at the relationship between suicidal ideation and the severity of depression, we used the depression module total score minus the score for the suicidal ideation item. ${ }^{1724-26}$ The suicidality item was excluded to avoid a spurious association between suicidal ideation and severity of depression. Odds ratios (OR) were computed with 95\% confidence intervals (CI). All analyses were performed by using SAS software (SAS Institute, Cary, NC). ${ }^{27}$

\section{Results \\ Rates of suicidal ideation and suicidality levels}

In total, 318 (15.4\%) of 2068 patients with chronic health conditions acknowledged any suicidal ideation. Among this group, $178(8.6 \%)$ reported only passive ideation, $76(3.7 \%)$ indicated active ideation without a plan, whereas $64(3.1 \%)$ admitted active ideation with a plan $(2.2 \%)$ or suicide attempt $(0.9 \%)$ in the past month. The remaining 1750 patients who did not report suicidal ideation constituted the nonsuicidal group.

\section{Sociodemographic and clinical characteristics}

Table 1 presents sociodemographic and clinical variables for the total sample, patients reporting any suicidal ideation, and the subgroup of suicide ideators classified by suicidality levels into four mutually exclusive groups, ie, passive ideation, active ideation without a plan, active ideation with a plan, and active ideation with an attempt.

The mean age of the patients with chronic illnesses participating in the assessment was 57 years. Half (50.8\%) had not completed high school, $75.9 \%$ were female, $54.2 \%$ were married, and approximately one quarter were employed. Over three quarters $(80.1 \%)$ of the patients reported moderate depression symptoms or less, $13.4 \%$ moderately severe depression symptoms, and $6.5 \%$ severe depression symptoms.

Patients with chronic health conditions who acknowledged any suicidal ideation were more likely than nonsuicidal patients to be female, younger, less educated, and unemployed. The groups also differed in rates of depression 


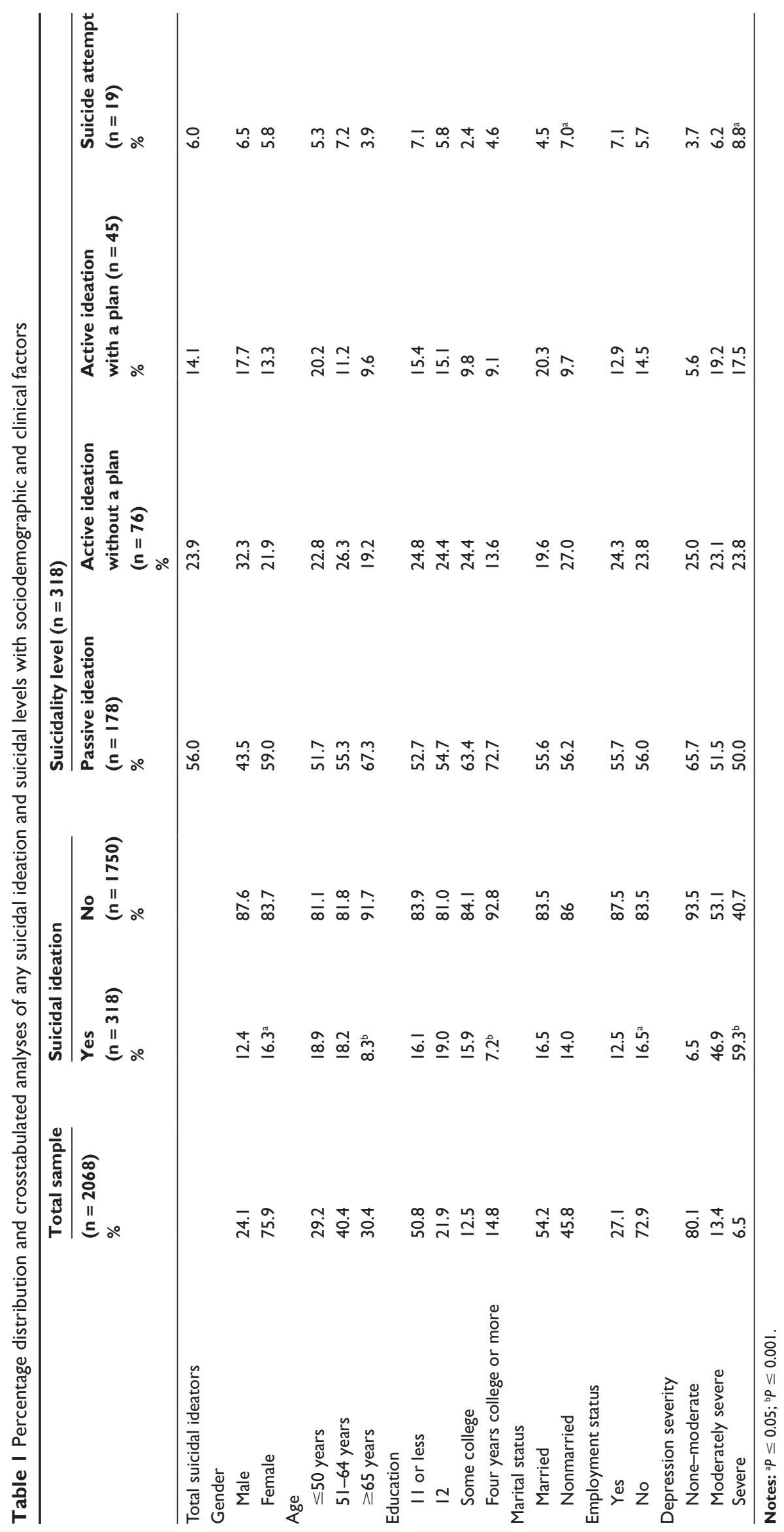


symptoms. Patients with higher levels of depression severity were found to have higher rates of suicidal ideation.

When we examined patients reporting suicidal ideation by suicidality level, we found that depression severity was significantly associated with suicidality risk level. Patients with higher depression severity were more likely to report higher levels of suicidality risk than patients with fewer depression symptoms. Most sociodemographic characteristics of the groups did not differ significantly.

\section{Sociodemographic and clinical correlates of any suicidal ideation}

Table 2 presents results from the logistic regression model fitted to the variables that were statistically significant or at least showed a trend toward significance in the bivariate analyses with suicidal ideation. Overall, the strongest risk for suicidal ideation among patients with chronic health conditions was depression severity level. The occurrence of suicidal ideation was 11 times higher (95\% CI: 8.4-15.7) among patients with moderately severe depression in comparison with patients with non or moderate depression symptoms. The risk was even higher for those reporting severe depression (OR $=18.8,95 \% \mathrm{CI}$ : 12.5-28.1). There was also evidence that age and education were the only sociodemographic variables that continued to be significantly associated

Table 2 Multiple logistic regression analysis of sociodemographic and clinical factors associated with suicidal ideation

\begin{tabular}{|c|c|c|}
\hline Variables & Odds ratio $(95 \% \mathrm{Cl})$ & $P$ value \\
\hline \multicolumn{3}{|l|}{ Gender } \\
\hline Male & 1 & \\
\hline Female & $1.04(0.74-1.48)$ & 0.811 \\
\hline \multicolumn{3}{|l|}{ Age } \\
\hline$\leq 50$ years & $1.74(1.39-2.65)$ & 0.011 \\
\hline $51-64$ years & $1.85(1.26-2.72)$ & 0.002 \\
\hline$\geq 65$ years & $\mathrm{I}$ & \\
\hline \multicolumn{3}{|l|}{ Education } \\
\hline $0-11$ & $2.21(1.31-3.74)$ & 0.003 \\
\hline 12 & $2.83(1.62-4.96)$ & 0.001 \\
\hline Some college & $2.32(1.25-4.34)$ & 0.008 \\
\hline Four years college or more & 1 & \\
\hline \multicolumn{3}{|l|}{ Marital status } \\
\hline Nonmarried & I & \\
\hline Married & $0.90(0.68-1.2 I)$ & 0.492 \\
\hline \multicolumn{3}{|l|}{ Employment status } \\
\hline Not employed & I & \\
\hline Employed & $0.82(0.58-1.16)$ & 0.264 \\
\hline \multicolumn{3}{|l|}{ Depression severity } \\
\hline None-moderate & I & \\
\hline Moderately severe & $11.46(8.36-|5.7|)$ & $<0.001$ \\
\hline Severe & 18.76 (12.53-28.09) & $<0.001$ \\
\hline
\end{tabular}

Abbreviation: $\mathrm{Cl}$, confidence interval. with suicidal ideation. Patients with chronic health conditions aged 64 years or younger were over one and a half times as likely to acknowledge suicidal ideation as those aged 65 years and older. Patients who reported that they had some years of college education or less demonstrated a twofold higher risk of suicidal ideation than those with four years of college or more.

\section{Sociodemographic and clinical correlates of suicidality severity levels}

The results of multinomial logistic regression models, classifying past-month suicidal ideation into three mutually exclusive levels of suicidal severity, ie, passive ideation (the reference group), active ideation without a plan, and active ideation with a plan or suicide attempt are presented in Table 3 . The only variable that was selected by this technique for the first outcome, active ideation without a plan in comparison with passive ideation was gender. Men were more likely to report active ideation without a plan than women.

While there was no difference in depression severity between patients with passive ideation and active ideation without a plan, depression severity was a significant risk for the second outcome, active ideation with a plan or suicide attempt compared with passive ideation. After adjusting for sociodemographic variables, depression severity was significantly associated with increased level of suicidality. Patients with severe depression symptoms were over three times $(\mathrm{OR}=3.56,95 \% \mathrm{CI}: 1.51-8.38)$ as likely as those with moderate or less depression symptoms to report active ideation with a plan or attempt.

\section{Discussion}

Results of this study show that suicidal ideation was elevated among our sample of patients with chronic health conditions in general medical practices in Puerto Rico, with 15.4\% acknowledging recent suicidal ideation. Congruent findings can be found among studies that examine suicidal ideation in the presence of a physical illness, for example, high rates are reported for patients with rheumatoid arthritis (11\%) and pulmonary disease $(11 \%-12 \%){ }^{28,11}$ It has been suggested that the presence of chronic physical illnesses may influence a person's mood or attitude towards life through fear of death, apprehension towards pain, or the perception that they are an unacceptable physical or economic burden on their families. ${ }^{2}$ Some illnesses may also induce clinical depression and increase the risk of suicidal behavior. Overall, the high rate of suicide ideation observed among our sample of patients with chronic physical illnesses is disturbing. 
Table 3 Multinomial logistic regression comparing two groups of active suicide ideators with passive ideators against sociodemographic and clinical factors

\begin{tabular}{|c|c|c|c|c|}
\hline \multirow[t]{2}{*}{ Variables } & \multicolumn{2}{|c|}{ Active ideation without plan } & \multicolumn{2}{|c|}{$\begin{array}{l}\text { Active ideation with plan or suicide } \\
\text { attempt }\end{array}$} \\
\hline & OR $(95 \% \mathrm{Cl})$ & $P$ value & OR $(95 \% \mathrm{Cl})$ & $P$ value \\
\hline \multicolumn{5}{|l|}{ Gender } \\
\hline Male & 1 & & 1 & \\
\hline Female & $0.45(0.23-0.89)$ & 0.021 & $0.55(0.26-1.15)$ & 0.113 \\
\hline Age & $0.98(0.96-1.01)$ & 0.207 & $0.98(0.95-1.00)$ & 0.092 \\
\hline Education & $0.96(0.90-1.03)$ & 0.238 & $0.98(0.90-1.05)$ & 0.515 \\
\hline \multicolumn{5}{|l|}{ Marital status } \\
\hline Nonmarried & 1 & & 1 & \\
\hline Married & $0.65(0.36-1.15)$ & 0.140 & $1.31(0.72-2.39)$ & 0.379 \\
\hline \multicolumn{5}{|l|}{ Employment status } \\
\hline Not employed & 1 & & 1 & \\
\hline Employed & $0.89(0.44-1.79)$ & $0.74 I$ & $0.88(0.4 I-1.87)$ & $0.74 I$ \\
\hline \multicolumn{5}{|l|}{ Depression severity } \\
\hline None-moderate & 1 & & 1 & \\
\hline Moderately severe & $1.20(0.63-2.26)$ & 0.581 & $3.58(1.62-7.93)$ & 0.002 \\
\hline Severe & $1.30(0.64-2.66)$ & 0.474 & $3.56(1.51-8.38)$ & 0.004 \\
\hline
\end{tabular}

Abbreviations: $\mathrm{Cl}$, confidence interval; OR, odds ratio.

Suicidal ideation was especially common in patients with high depressive symptoms. Our findings confirm that depression severity is a significant risk factor for suicidal ideation. Over $40 \%$ of the patients reporting moderately severe depression symptoms reported suicidal ideation, while almost $60 \%$ of those with severe depression symptoms acknowledged suicidal ideation. These findings are similar to those of studies in other patient groups in the US and other countries, showing that the severity of depressive symptoms are among the most frequent and strongest correlates of suicidal ideation. ${ }^{29-31}$

Suicidal ideation also varied by age groups, in that patients aged 64 years or younger with chronic illnesses tended to report higher rates of suicidal ideation than older patients. This age difference may represent a cohort effect, whereby older patients might be more prepared to accept and deal with the impact of chronic health conditions. It is also possible that older patients have protective social and cultural factors that act to mitigate the effects of exposure to risk factors for suicidal ideation. Studies of suicidal behaviors among older adults suggest there is a range of potential protective factors, including social support, social connectedness and social interaction, strong religious and spiritual values, and unfavorable attitudes toward suicide, among others. ${ }^{32,33}$ Additional research is needed to identify the potential factors that may contribute to protect older patients in our study population.

Another interesting finding was that even after adjusting for potential confounders, suicidal ideation was two times higher among patients without a college degree in comparison with those who had obtained a college degree. Other research has indicated that the risks of suicide and suicide attempt are elevated among individuals with less education. ${ }^{32,33}$ It has been suggested that the major route by which educational attainment may influence suicidal ideation is as a result of links between socioeconomic factors and mental health. ${ }^{34}$ Beautrais et al point out that the detection and treatment of depression may be important factors in preventing suicide among less educated patients with chronic health conditions..$^{35}$

A further aim of this study was to evaluate the severity of suicidal ideation among our study sample of patients with chronic health conditions and to identify variables that might be associated with suicidal ideation. Until effective interventions for suicide prevention are developed, identifying populations at increased risk for suicide is essential. Learning about the risk or protective factors separating passive suicidal ideation from more serious ideation could contribute to identifying those with increased need of further clinical assessment and preventive measures.

Among the 318 patients who acknowledged recent suicidal ideation, almost half reported active ideation (20.1\% with a plan or recent attempt and $23.9 \%$ without a plan), whereas the others $(56 \%)$ reported passive suicidal ideation. Consistent with results from other studies we found a strong relationship between depression severity and the level of self-harm reported in the ideation..$^{24,36}$ Suicide ideators with severe depression were over three times more likely to have 
a suicide plan or attempt than those with lower depression symptoms. These findings suggest that increased depression severity appears to be a significant risk factor for having a suicidal plan or attempt, and thus most likely for suicide.

Similar to findings with other ethnic/racial groups, this study in general practice patients in Puerto Rico provides strong evidence supporting the predominant role of depression severity in increasing the severity of suicidal ideation. ${ }^{24,31,36,37}$ The high prevalence rate of active suicidal ideation among patients with chronic illnesses, as well as its strong link with depression severity highlights the importance of reducing suicidal behavior among this group.

Early identification and effective treatment of depression may be the most effective measure for preventing suicidal behavior. The "National Strategy for Suicide Prevention", a report that articulates a framework to guide national action in the prevention of suicide, noted that improving the management of depression is fundamental, given that this disorder is implicated in a high proportion of suicides. ${ }^{38}$ Depression is frequently experienced by patients receiving care in the primary care setting. ${ }^{39}$ In 2002, the US Preventive Services Task Force recommended that routine screening be conducted in primary care to improve depression detection rates. ${ }^{40}$ Studies reviewed evidenced better clinical outcomes when screening was connected with systemic efforts to ensure adequate treatment and follow-up. As such, the recommendation highlighted that screening be conducted only in practices that have appropriate systems in place to effectively diagnose, treat, and provide follow-up care.

The American College of Preventive Medicine issued a position paper in which they manifest their support for this recommendation and elaborate on how primary care settings could put in place appropriate systems to ensure the accurate diagnosis and treatment of depression. ${ }^{41}$ They provide details on standardized depression screening measures and discuss relevant information to be considered in the selection process. Screening measures provide an initial assessment that should be followed by an assessment that examines relevant criteria for diagnosis, including symptom duration, level of impairment, and comorbid substance use or psychiatric disorders. The use of standardized treatments with established efficacy is supported. Symptom remission is identified as the targeted endpoint. Such a system could be established within the primary care setting or in collaboration with specialized mental health settings. Guidelines issued by the Institute for Clinical Systems Improvement also point to the importance of providing education that promotes patient engagement and participation, as well as maintaining ongoing contact with patients during the first 6-12 months of treatment to facilitate follow-up care and tracking of treatment response. ${ }^{42}$

In 2009, an updated systematic review of depression trials allowed incorporation of more recent intervention studies published since the previous review. ${ }^{43}$ Larger benefits were observed in programs that combined depression screening with the assistance of additional staff who provided some part of the depression care, such as care management, or made additional efforts to facilitate that patients use specialty mental health treatment. Reduced benefits were evidenced when staff support was not accessible. The authors of the updated review commented that these results were not surprising given the amount of work required from the primary care clinician. Review findings led the US Preventive Services Task Force to update its 2002 recommendation specifying that adult depression screening in primary care also needed "staff-assisted depression care supports" to be in place to assure accurate diagnosis, effective treatment, and follow-up. ${ }^{44}$

This study has some limitations that should be considered when interpreting its findings. First, we analyzed data from patients with different chronic physical conditions together; associations with suicidal ideation that were unique to a particular group may have been diluted. Although it would be preferable to examine such groups, limited data precluded this effort. Second, we had only information on the presence or absence of chronic physical conditions rather than their severity, while the latter may be a significant contributor to the patient's suicidal risk level. Third, representativeness is another shortcoming. The data presented were collected from a convenience sample of adults receiving care at general practices located in urban settings serving mainly lower socioeconomic patients. Fourth, the problem of bias due to social desirability and stigma is a well known occurrence in these kind of studies. Data were collected by face-to-face interviews to help reduce nonparticipation. However, underreporting may result due to respondents' reluctance to admit suicidal thoughts or behaviors. We cannot precisely determine the extent to which underreporting biased our results but, if this was the case, the rates of suicidal ideation would be even higher than those reported. On the other hand, to avoid an overestimation of active suicidal ideation, a major strength of this study is the two-tier assessment strategy used for assessing suicidality. Patients endorsing an initial question of suicidal ideation were further questioned to identify the severity of suicidal ideation, allowing us to make a distinction between passive ideation, active ideation without a plan, and active ideation with a plan or suicide attempt. 


\section{Conclusion}

To the best of our knowledge. no other study has assessed rates, severity, and correlates of suicidal ideation among general practice patients with chronic health conditions in Puerto Rico. Several significant findings were identified. First, our results evidence elevated rates of suicidal ideation among our study group. Another very important finding was that patients with severe depression had the highest likelihood of high-risk suicidal ideation compared with those with moderate or no depression. Consistent with research findings in other ethnic/racial populations, our results show a strong link between depression severity and suicidal risk. These findings suggest that public health treatment strategies focusing on the systematic identification of depressed patients and the implementation of appropriate systems to ensure the accurate diagnosis and treatment of depression in general medical settings are relevant for minimizing and preventing suicidal behavior among patients with chronic health conditions.

\section{Acknowledgments}

This project was supported by grant SC1MH090599 from the National Institute of General Medical Sciences.

\section{Disclosure}

The authors report no conflicts of interest in this work.

\section{References}

1. WHO Health Organization. World Report on Violence and Health. Geneva, Switzerland: World Health Organization; 2002.

2. Moscicki EK. North American perspectives: Epidemiology of suicide. Int Psychogeriatr. 1995;7(2):137-148.

3. Saraceno B. WHO perspective on mental health beyond 2001: Putting the evidence into action. In: Vijayahumar L, editor. Suicide Prevention: Meeting the Challenge Together. Hyderabad, India: Editorial Orient Longman Pvt Ltd; 2003.

4. World Health Organization. Preventing Suicide, Mental and Behavioural Disorders. Geneva, Switzerland: World Health Organization; 2000.

5. Baldessarini RJ, Tondo L, Hennen J. Lithium treatment and suicide risk in major affective disorders: Update and new findings. J Clin Psychiatry. 2003;64 Suppl 5:44-52.

6. Wilson I, Duszynski K, Mant A. A 5-year follow-up of general practice patients experiencing depression. Fam Pract. 2003;20(6):685-689.

7. US Public Health Service. The Surgeon General's Call To Action To Prevent Suicide. Washington, DC: US Public Health Service; 1999.

8. Institute of Medicine. Reducing Suicide: A National Imperative. Washington, DC: National Academy of Sciences; 2002.

9. Brown ES, Khan DA, Mahadi S. Psychiatric diagnoses in inner city outpatients with moderate to severe asthma. Int J Psychiatry Med. 2000; 30(4):319-327.

10. Druss B, Pincus H. Suicidal ideation and suicide attempts in general medical illnesses. Arch Intern Med. 2000;160(10):1522-1526.

11. Goodwin RD, Kroenke K, Hoven CW, Spitzer RL. Major depression, physical illness, and suicidal ideation in primary care. Psychosom Med. 2003;65(4):501-505.
12. Goodwin RD, Marusic A, Hoven CW. Suicide attempts in the United States: The role of physical illness. Soc Sci Med. 2003;56(8): $1783-1788$.

13. Kishi Y, Robinson RG. Suicidal plans following spinal cord injury: Six month study. J Neuropsychiatry Clin Neurosci. 1996;8(4):442-445.

14. Kishi Y, Robinson RG, Kosier JT. Suicidal ideation among patients with acute life-threatening physical illness: Patients with stroke, traumatic brain injury, myocardial infarction, and spinal cord injury. Psychosomatics. 2001;42(5):382-390.

15. Angst J, Angst F, Stassen HH. Suicide risk in patients with major depressive disorder. J Clin Psychiatry. 1999;60 Suppl 2:57-62.

16. De Leo D, Spatonis K. Suicidal behavior. In: Griez EJL, Faravelu C, Nutt DJ, Zohar J, editors. Mood Disorder: Clinical Management and Research Issues. New York, NY: John Wiley \& Sons, Ltd.; 2005.

17. Szanto K, Mulsant BH, Houck P, Dew MA, Reynolds CF 3rd. Occurrence and course of suicidality during short-term treatment of late-life depression. Arch Gen Psychiatry. 2003;60(6):610-617.

18. Schulberg HC, Lee PW, Bruce ML, et al. Suicidal ideation and risk levels among primary care patients with uncomplicated depression. Ann Fam Med. 2005;3(6):523-528.

19. Vera M, Perez-Pedrogo C, Huertas SE, et al. Collaborative care for depressed patients with chronic medical conditions: A randomized trial in Puerto Rico. Psychiatr Serv. 2010;61(2):144-150.

20. Spitzer RL, Kroenke K, Williams JB. Validation and utility of a selfreport version of PRIME-MD: The PHQ primary care study. Primary Care Evaluation of Mental Disorders. Patient Health Questionnaire. JAMA. 1999;282(18):1737-1744.

21. Sheehan DV, Lecrubier Y, Sheehan KH, et al. The Mini-International Neuropsychiatric Interview (M.I.N.I.): The development and validation of a structured diagnostic psychiatric interview for DSM-IV and ICD-10. J Clin Psychiatry. 1998;59 Suppl 20:22-33.

22. Kroenke K, Spitzer RL, Williams JB. The PHQ-9: Validity of a brief depression severity measure. J Gen Intern Med. 2001;16(9):606-613.

23. Huang FY, Chung H, Kroenke K, Delucchi KL, Spitzer RL. Using the Patient Health Questionnaire-9 to measure depression among racially and ethnically diverse primary care patients. J Gen Intern Med. 2006; 21(6):547-552.

24. Alexopoulos GS, Bruce ML, Hull J, Sirey JA, Kakuma T. Clinical determinants of suicidal ideation and behavior in geriatric depression. Arch Gen Psychiatry. 1999;56(11):1048-1053.

25. Casey PR, Dunn G, Kelly BD, et al. Factors associated with suicidal ideation in the general population: Five-centre analysis from the ODIN study. Br J Psychiatry. 2006;189:410-415.

26. O'Leary CC, Frank DA, Grant-Knight W, et al. Suicidal ideation among urban nine and ten year olds. J Dev Behav Pediatr. 2006;27(1):33-39.

27. SAS/STAT 9.1 User's Guide. Cary, NC: SAS Institute Inc.; 2004.

28. Treharne GJ, Lyons AC, Kitas GD. Suicidal ideation in patients with rheumatoid arthritis. Research may help identify patients at high risk. BMJ. 2000;321(7271):1290.

29. Bartels SJ, Coakley E, Oxman TE, et al. Suicidal and death ideation in older primary care patients with depression, anxiety, and at-risk alcohol use. Am J Geriatr Psychiatry. 2002;10(4):417-427.

30. Pfaff JJ, Almeida OP. Detecting suicidal ideation in older patients: Identifying risk factors within the general practice setting. Br J Gen Pract. 2005;55(513):269-273.

31. Vuorilehto MS, Melartin TK, Isometsa ET. Suicidal behaviour among primary-care patients with depressive disorders. Psychol Med. 2006;36(2):203-210.

32. Beautrais AL. Suicides and serious suicide attempts: Two populations or one? Psychol Med. 2001;31(5):837-845.

33. Lesage AD, Boyer R, Grunberg F, et al. Suicide and mental disorders: A case-control study of young men. Am J Psychiatry. 1994;151(7): 1063-1068.

34. Fergusson DM, Lynskey MT. Childhood circumstances, adolescent adjustment, and suicide attempts in a New Zealand birth cohort. $J$ Am Acad Child Adolesc Psychiatry. 1995;34(5):612-622. 
35. Beautrais AL, Collings SCD, Ehrhardt P. Suicide prevention: A review of evidence of risk and protective factors, and points of effective intervention. Wellington, New Zealand: Ministry of Health; 2005. Available at: http:// www.moh.govt.nz/moh.nsf/pagesmh/4086. Accessed January 10, 2011.

36. Pages KP, Russo JE, Roy-Byrne PP, Ries RK, Cowley DS. Determinants of suicidal ideation: The role of substance use disorders. J Clin Psychiatry. 1997;58(11):510-515.

37. Gaynes BN, Rush AJ, Trivedi MH, et al. Major depression symptoms in primary are and psychiatric care settings: A cross-sectional analysis. Ann Fam Med. 2007;5(2):126-134.

38. US Dept of Health and Human Services. National Strategy for Suicide Prevention: Goals and Objectives for Action. Rockville, MD: Public Health Service; 2001.

39. Shim RS, Baltrus P, Ye J, Rust G. Prevalence, treatment, and control of depressive symptoms in the United States: Results from the National Health and Nutrition Examination Survey (NHANES), 2005-2008. J Am Board Fam Med. 2011;24(1):33-38.
40. US Preventive Services Task Force. Screening for depression Recommendations and rationale. Ann Intern Med. 2002;136(10): 760-764.

41. Nimalasuriya K, Compton MT, Guillory VJ. Screening adults for depression in primary care: A position statement of the American College of Preventive Medicine. J Fam Pract. 2009;58(10):535-538.

42. Institute for Clinical Systems Improvement. Health care guideline: Major depression in adults in primary care. 2010. Available at: http:// www.icsi.org/guidelines_and_more/gl_os_prot/behavioral_health/ depression_5/depression_major_in_adults_in_primary_care_4.html. Accessed January 18, 2011.

43. O’Connor EA, Whitlock EP, Beil TL, Gaynes BN. Screening for depression in adult patients in primary care settings: A systematic evidence review. Ann Intern Med. 2009;151(11):793-803.

44. US Preventive Services Task Force. Screening for depression in adults: US Preventive Services Task Force recommendation statement Ann Intern Med. 2009;151(11):784-792.
International Journal of General Medicine

\section{Publish your work in this journal}

The International Journal of General Medicine is an international, peer-reviewed open-access journal that focuses on general and internal medicine, pathogenesis, epidemiology, diagnosis, monitoring and treatment protocols. The journal is characterized by the rapid reporting of reviews, original research and clinical studies across all disease areas.

\section{Dovepress}

A key focus is the elucidation of disease processes and management protocols resulting in improved outcomes for the patient.The manuscript management system is completely online and includes a very quick and fair peer-review system. Visit http://www.dovepress.com/ testimonials.php to read real quotes from published authors.

Submit your manuscript here: http://www.dovepress.com/international-journal-of-general-medicine-journal 